\title{
Perfil de manipuladores de alimentos em domicílios
}

\author{
Profile of food handlers in the home
}

Barbara Cecconi Deon ${ }^{1}$

Laissa Benites Medeiros ${ }^{1}$

Luisa Helena Hecktheuer ${ }^{1}$

Ana Lúcia de Freitas Saccol ${ }^{1}$

${ }^{1}$ Departamento de Tecnologia e Ciência dos Alimentos, Centro de Ciências Rurais, Universidade Federal de Santa Maria. Campus Universitário Sala 3211, Camobi. 97.105-900 Santa Maria RS Brasil.

bcdeon@hotmail.com
Abstract This article seeks to diagnose the profile of food handlers in the households of the city of Santa Maria in the state of Rio Grande do Sul. By geographical division of the city, a sample of 15 households per neighborhood was chosen, totaling 615 households. For the diagnosis of the profile of handlers in the households, a questionnaire was prepared, containing 6 open questions and 13 closed questions related to personal data including gender, marital status, education, occupation and monthly income. Issues such as the frequency with which people prepare meals in the households and satisfaction in preparing them, as well as participation in courses and the perception of food safety, were also addressed. It was found that $92.5 \%$ of the food handlers were female, $52.2 \%$ had complete or incomplete primary school and $35.4 \%$ had a monthly income of between 3 and 9 minimum wages. Among those interviewed, $82.6 \%$ reported that they prepare meals every day of the week and $77.9 \%$ reported that they never had problems caused by the food produced at home. It is important to evaluate the profile of food handlers in the households, as this makes it possible to develop strategies to prevent or diminish foodborne diseases.

Key words Food handler, Food safety, Population characteristics, Foodborne diseases
Resumo O objetivo deste artigo é identificar o perfil dos manipuladores de alimentos nos domicílios da cidade de Santa Maria (RS). Foi determinada uma amostra de 15 domicílios por bairro através da divisão geográfica da cidade, totalizando 615 residências. Para a realização do diagnóstico do perfil dos manipuladores nos domicílios, elaborou-se um formulário sobre os dados pessoais com 6 questões abertas e 13 fechadas incluindo o gênero, estado civil, escolaridade, profissão e renda mensal, bem como questões como a frequência do preparo das refeições nos domicílios, satisfação em prepará-las, participação em cursos e percepção deles sobre a segurança dos alimentos. Verificou-se que 92,5\% dos manipuladores eram do sexo feminino, 52,2\% possuíam nivel de escolaridade fundamental completo ou incompleto, e $35,4 \%$ com rendimento mensal familiar de 3 a 9 salários mínimos. Dos entrevistados, $82,6 \%$ informaram que preparam as refeições todos os dias da semana e 77,9\% relataram que nunca enfrentaram problemas ocasionados por alimentos produzidos em casa. É importante identificar o perfil dos manipuladores de alimentos nos domicílios para desenvolver estratégias para prevenir ou diminuir as doenças transmitidas pelos alimentos. Palavras-chave Manipulador de alimentos, Segurança dos alimentos, Características da população, Doenças transmitidas por alimentos 


\section{Introdução}

De acordo com a Resolução da Diretoria Colegiada (RDC) no 216 de 15 de setembro de 2004 da Agência Nacional de Vigilância Sanitária (ANVISA), que dispõe de regulamento técnico de Boas Práticas para Serviços de Alimentação, o manipulador de alimentos é definido como qualquer pessoa que entra em contato direto ou indireto com o alimento ${ }^{1}$.

O termo "manipulador de alimentos" corresponde a qualquer indivíduo que entre em contato com um produto alimentício nas etapas de recepção, produção, processamento, embalagem, armazenamento e venda de alimentos. São pessoas que podem contaminar os alimentos, provocando Doenças Transmitidas por Alimentos (DTA), quando aplicam técnicas incorretas na produção de refeições, na higienização de equipamentos, de utensílios e do próprio ambiente $^{2,3}$; entre outros procedimentos que propiciem a contaminação, a multiplicação e a sobrevivência de microrganismos patogênicos.

No Brasil, segundo dados da Análise Epidemiológica dos Surtos de DTA, no período de 2000 a 2011, 51,8\% (3746 casos) dos locais de ocorrência dos surtos tiveram origem domiciliar, estando acima de todos os outros locais, inclusive restaurantes ${ }^{4}$.

De acordo com a Autoridade Europeia de Segurança dos Alimentos ${ }^{5}$, $95 \%$ dos casos de intoxicação alimentar são devidos a pequenos focos que se originam em casa. Estudos confirmam estes dados, como Redmond e Griffith ${ }^{6}$, que constataram 50\% das ocorrências de DTA nos domicílios, e Van Asselt et al. ${ }^{7}$, que indicaram até $87 \%$. Dos surtos de gastroenterite para o período entre 1998 e 2000 na Nova Zelândia, 39,3\% dos casos foram adquiridos em casa ${ }^{8}$.

Várias pesquisas têm sido feitas sobre os conhecimentos, as atitudes e as práticas dos manipuladores de alimentos ${ }^{9-12}$. De acordo com Oliveira et al. ${ }^{2}$, o perfil higiênico-sanitário dos manipuladores de alimentos tem se mostrado frequentemente inaceitável no que diz respeito à contaminação microbiana encontrada em diversos sítios anatômicos como nariz e boca. Meldrum et al. ${ }^{13}$ e Sagoo et al. ${ }^{14}$ verificaram que as práticas de manipulação de alimentos nas residências propiciam o crescimento microbiano.

Sanlier ${ }^{15}$ afirma que o manipulador não tem conhecimento suficiente sobre higiene pessoal, preparação e práticas de armazenamento de alimentos e que muitos consumidores não têm sequer informações básicas sobre detergentes, de- sinfetantes, esterilização ou agentes prejudiciais. Estudos revelaram que os consumidores não têm noção de como comprar, preparar, cozinhar e armazenar leite, alimentos enlatados, carne, etc., e que eles não possuem conhecimento da existência de microrganismos nos alimentos e não dão atenção à higiene pessoal e do seu ambiente enquanto preparam alimentos ${ }^{15-18}$.

Redmond e Griffith ${ }^{19}$ destacam que, de forma geral, os manipuladores possuem conhecimento inadequado a respeito da natureza e da origem das DTA, subestimando suas consequências. Oliveira et al. ${ }^{2}$ acrescentam que essas doenças em decorrência de contaminação microbiana têm origem na ignorância e no descaso dos manipuladores. Sendo assim, esses fatores podem ser uma importante barreira para as mudanças de comportamento e a adoção de práticas seguras de higiene dos alimentos ${ }^{19,20}$.

Neste sentido, ressalta-se a necessidade de conhecer melhor os manipuladores de alimentos nos domicílios, para possibilitar o desenvolvimento de estratégias específicas para prevenção das DTA nestes locais. Com base no exposto, o objetivo deste estudo foi diagnosticar o perfil dos manipuladores de alimentos nos domicílios da cidade de Santa Maria (RS).

\section{Métodos}

Para desenvolver a pesquisa, uma amostra de domicílios da cidade de Santa Maria (RS) foi selecionada através da atual divisão geográfica da cidade. Pela lei complementar $n^{\circ} 042$, de 29 de dezembro de 2006, a divisão geográfica da zona urbana de Santa Maria está representada por 8 Regiões Administrativas: Centro Urbano; Norte; Nordeste; Leste; Centro-Leste; Sul; Centro-Oeste e Oeste. Cada uma, por sua vez, está subdividida em unidades de vizinhança, denominadas "bairro", ligando entre si as Unidades Residenciais com características urbanas semelhantes. Dessa forma, a cidade contém 41 bairros distribuídos nas 8 Regiões Administrativas ${ }^{21}$. O presente estudo foi desenvolvido no período de maio a agosto de 2011.

Uma amostra probabilística de 15 domicílios para cada bairro foi determinada, totalizando-a em 615 residências. Como critérios de inclusão, considerou-se o interesse e a disponibilidade do responsável pela manipulação em participar da pesquisa mediante assinatura do Termo de Consentimento Livre e Esclarecido. Não foram selecionados os domicílios em que o manipula- 
dor de alimento, no momento da entrevista, não se encontrava na residência.

Para a realização do diagnóstico do perfil dos manipuladores nos domicílios, elaborou-se um formulário incluindo dados pessoais como gênero, data de nascimento, estado civil, nível de escolaridade, profissão, renda mensal domiciliar, número de pessoas residentes nos domicílios e acesso aos meios de comunicação; bem como questões como a frequência do preparo das refeições nos domicílios, satisfação em preparar as refeições, participação em cursos na área de alimentos, e percepção dos manipuladores em relação à segurança dos alimentos, totalizando 6 questões abertas e 13 fechadas. O formulário foi previamente testado e aplicado por pessoas técnicas e capacitadas para tal finalidade; a aplicação foi na forma de entrevista face a face e a busca dos domicílios em cada bairro foi aleatória.

Os dados referentes ao presente estudo foram analisados através do software Statistical Package for Social Science 15.0 (SPSS) no qual foram utilizadas estatísticas descritivas simples de frequência (média e desvio padrão). Esta pesquisa foi aprovada em seus aspectos éticos e metodológicos pelo Comitê de Ética em Pesquisa da Universidade Federal de Santa Maria (RS) e reconhecido pela Comissão Nacional de Ética em Pesquisa, do Ministério da Saúde, através do Certificado de Apresentação para Apreciação Ética (CAAE).

\section{Resultados e discussão}

Dos 615 manipuladores de alimentos nos domicílios da cidade de Santa Maria (RS), observouse que $92,5 \%$ eram do gênero feminino, demonstrando que ainda a mulher é a responsável por preparar e organizar a alimentação na família. Concordando com esta pesquisa, Losasso et al. ${ }^{22}$ verificaram que $90 \%$ dos responsáveis por manipular os alimentos em suas residências são mulheres. $\mathrm{Szabo}^{23}$ afirma que a culinária doméstica é uma tarefa essencialmente feminina.

Uma pesquisa realizada pelo Instituto Brasileiro de Geografia e Estatística mostrou que a crescente participação das mulheres no mercado de trabalho não reduziu a jornada delas com os afazeres domésticos. Pelo contrário, na faixa etária de 25 a 49 anos de idade, na qual a inserção das mulheres nas atividades remuneradas é maior e coincide com a presença de filhos menores, $\mathrm{o}$ trabalho doméstico ocupa $94,0 \%$ das mulheres. Corroborando com os dados do presente estu- do, no país, 109,2 milhões de pessoas de 10 anos ou mais de idade declararam realizar tarefas domésticas, sendo que, deste conjunto, 71,5 milhões (65,4\%) são mulheres ${ }^{24}$.

Avaliou-se o estado civil dos entrevistados e verificou-se que $62,6 \%$ das pessoas entrevistadas eram casadas. Gong et al. ${ }^{25}$ constataram que $58,8 \%$ dos manipuladores de alimentos dos domicílios de cidades da China eram mulheres; e, entre estas, 58,4\% eram casadas, concordando com os achados deste estudo.

Quando avaliada a idade dos participantes, constatou-se que a média foi 53 anos $\pm 16,10 \mathrm{DP}$ (desvio padrão), tendo como mínimo de 18 e máximo de 89 anos. O perfil dos entrevistados foi semelhante ao estudo realizado por Losasso et al..$^{22}$, em contrapartida, difere de Gong et al..$^{25}$ por mostrarem que a idade média dos participantes do seu estudo em domicílios foi entre $18 \mathrm{e}$ 35 anos, correspondendo ao percentual de 55\% da amostra pesquisada.

Soares e Sabóia ${ }^{24}$, na Pesquisa Nacional por Amostra de Domicílios de 2001 e 2005, verificaram que os homens acima de 60 anos dedicavam parte do seu tempo em afazeres domésticos (13 horas semanais). Já para as mulheres, o trabalho doméstico se intensifica a partir dos 50 anos de idade, mas consome mais tempo das mulheres de 50 a 59 anos de idade ( 31 horas semanais), cerca de 3 vezes mais do que o tempo dedicado pelos homens.

Verificou-se que mais da metade dos entrevistados $(52,2 \%)$ possuíam nível de escolaridade fundamental completo ou incompleto. Dados da Pesquisa de Orçamento Familiar do período de 2008 a 2009 informaram que 47,41\% dos domiciliados do Brasil possuem somente ensino fundamental ${ }^{26}$.

Pode-se avaliar que o alto nível de escolaridade é inversamente proporcional aos afazeres domésticos, ou seja, foi menor o número de entrevistados exercendo tarefas domésticas com alto nível de escolaridade. Isso pode ser verificado, pois apenas 14,8\% dos entrevistados tinham nível de escolaridade superior completo ou incompleto, ou pós-graduação completa ou incompleta.

Corroborando com esta pesquisa, Soares e Sabóia ${ }^{24}$ constataram que a variável escolaridade exerce um papel interessante na análise do tempo de ocupação doméstica. Quando analisaram a jornada média de afazeres domésticos, averiguaram que para a população com 12 anos ou mais de estudos, o tempo dedicado aos serviços domésticos foi menor, sejam homens ou mulheres. Isso ocorre porque a escolaridade tem uma 
alta correlação com o rendimento, permitindo a contratação de outras pessoas para desempenhar essas tarefas. Para as mulheres com o nível de escolaridade maior, a jornada com o trabalho doméstico foi de cerca de 5 horas por semana a menos do que a observada para aquelas menos escolarizadas.

Quando questionados a respeito da profissão, 63,9\% dos entrevistados informaram exercer a profissão do lar. Esse percentual justifica-se, pois no momento do diagnóstico essas pessoas estavam presentes nos seus domicílios. Considerou-se do lar, também, as pessoas aposentadas, mas que no momento estavam desempenhando o papel de donas de casa. Foram relatadas pelos manipuladores profissões diversas, como: secretária, agente de saúde, massoterapeuta, advogado, porteiro, entre outros; todas essas profissões com horários variados de trabalho, por isso encontravam-se em suas residências durante a coleta das informações nos domicílios.

A renda mensal familiar foi avaliada e constatou-se que a maior frequência do rendimento mensal familiar correspondeu de 3 a 9 salários mínimos (35,4\%), ou seja, entre R \$ 1635,00 e R \$ 4905,00 (salário mínimo de referência: $\mathrm{R} \$ 545,00$ ). Os resultados do Censo Demográfico (2010) mostram que a desigualdade de renda ainda é bastante acentuada no Brasil. A média nacional de rendimento domiciliar per capita é de $\mathrm{R} \$ 668,00$ no país, com uma média de 3,34 integrantes por domicílio ${ }^{27}$.

Segundo dados da Pesquisa de Orçamento Familiar (POF) brasileira, do período de 2008 a 2009, o rendimento total médio mensal auferido foi de $\mathrm{R} \$ 2641,63$ e, agregando-se à variação patrimonial, este valor alcançou, em média, R\$ 2763,47. Na região sul do país apresenta-se um rendimento médio mensal familiar de $\mathrm{R} \$ 2873,52$; o que corresponde a aproximadamente 5 salários mínimos ${ }^{27}$.

A variável renda domiciliar merece destaque, pois está relacionada ao número de pessoas que residem no domicílio. Neste estudo, constatou-se que $56,6 \%$ dos entrevistados referiram obter até 3 salários mínimos, um montante menor que $\mathrm{R} \$$ 1635,00 para cobrir todas as despesas familiares, sendo que $58,9 \%$ dos manipuladores domésticos relataram que residem com até 3 pessoas, 38,3\% entre 4 e 6 , e 2,8\% acima de 6 pessoas.

Verificou-se que $82,6 \%$ das pessoas entrevistadas informaram que preparam as refeições todos os dias da semana. Como já era esperado, $67,2 \%$ dos manipuladores confirmaram que preparam somente o almoço, enquanto os demais,
$9,3 \%$ relataram o jantar e $23,6 \%$ ambas as refeições. Existe uma tendência do aumento do consumo de alimentos fora do lar, como refere a pesquisa de Akutsu et al. ${ }^{28}$, a qual estima que no Brasil a cada cinco refeições uma é feita fora de casa, enquanto na Europa são duas em cada seis e nos Estados Unidos uma em cada duas.

Quanto à satisfação dos manipuladores em relação à preparação dos alimentos nos domicílios, $82,4 \%$ relataram gostar de preparar as refeições e $88,3 \%$ deles gostariam de aprender sobre manipulação de alimentos seguros. Avaliar a satisfação dos manipuladores dos domicílios é um fator importante, pois se pode compreender a motivação e o empenho dos mesmos durante o preparo das refeições. Em contrapartida, devese avaliar as fragilidades relatadas pelos manipuladores domésticos quando foram informadas insatisfações durante o preparo das refeições. Dentre as respostas salientadas apontamse: "preparar as refeições é como uma obrigação", "ter pouca variedade nas preparações", "pouco tempo", "faz muita sujeira". Esta reflexão fornece subsídios para programas educativos junto a este público alvo.

Daniels et al. ${ }^{29}$ constataram que as pessoas consideram cozinhar principalmente como uma tarefa doméstica que precisa ser feita. Em média, metade do tempo gasto na preparação dos alimentos pelos participantes é motivada por necessidade. Entretanto, cozinhar também tende a ser um ato prazeroso ou agradável em menor grau, pois demonstra cuidado com as outras pessoas.

Em relação à participação em cursos na área de alimentos, observou-se que apenas $17,2 \%$ responderam ter participado de algum curso como: preparação de tortas, doces e salgados, pães e folhados. A maioria dos responsáveis pela manipulação de alimentos nos domicílios avaliados nunca recebeu qualquer instrução quanto à manipulação segura de alimentos. Wilcock et al. ${ }^{30}$ afirmam que a maioria das pessoas que preparam alimentos nos domicílios tem noções básicas de segurança, no entanto, nem sempre pratica estes comportamentos.

Quando questionados se algum membro da família já havia enfrentado algum problema ocasionado pelos alimentos produzidos em casa, um percentual expressivo, de $77,9 \%$, respondeu que não. Shapiro et al. ${ }^{31}$ também verificaram que os manipuladores de alimentos dos domicílios acreditavam que essa tarefa, quando realizada em casa, não é uma causa de DTA.

Uma explicação para este alto percentual pode ser o fato de que a maioria das pessoas associa a 
contaminação dos alimentos ao consumo fora do lar. Esse fato foi confirmado pela pesquisa de Leite et al. ${ }^{32}$, os quais avaliaram a percepção de donas de casa em relação ao risco de contrair DTA por consumir alimentos preparados nos domicílios. Constataram resultados semelhantes a este estudo, no qual 77,5\% dos entrevistados acreditavam que o risco de contrair DTA em suas residências era baixo ou inexistente, enquanto $70,0 \%$ acreditavam que esse risco era alto para os alimentos consumidos fora de casa ${ }^{32}$.

Dados comprovam a ocorrência de surtos de DTA em domicílios no Brasil. Estudo realizado por Leite e Waissmann ${ }^{33}$ mostra que no período de 2000 a 2002 ocorreram 42\% (147) de surtos de DTA de origem domiciliar no Brasil. Confirmando estes dados, Welker et al. ${ }^{34}$ verificaram no mesmo país, no período de 2006 a 2007, que 43\% das ocorrências foram notificadas nas residências e que $37 \%$ foram causados pela Samolnella ssp.

Em um município no estado de Alagoas, no Brasil, ocorreu um surto de botulismo em um domicílio acometendo cinco pessoas, tendo como possível causa a germinação de esporos de Clostridium botulinum na sardinha em molho de tomate picante e na mortadela de frango ${ }^{35}$.

Em outro município no estado de Pernambuco, no mesmo país, foi confirmada a ocorrência de um surto envolvendo arroz branco, frango frito, batata palha e tomate produzidos em uma residência, sendo que a causa mais provável de contaminação foi por manipulação sem higienização das mãos e exposição prolongada sem armazenamento adequado ${ }^{36}$.

Quando questionados a respeito dos meios de comunicação, 75,1\% dos entrevistados relataram ter acesso a mais de 2 meios de comunicação como televisão, rádio, jornal, Internet e revista. Isto demonstra que existe uma boa acessibilidade dos meios de comunicação pelos manipuladores de alimentos nos domicílios, uma vez que isso pode servir de modelo para campanhas informativas que envolvam a segurança dos alimentos nos municípios, pois as informações podem ser transmitidas para um grande público, e não necessariamente somente à amostra pesquisada. Unusan ${ }^{37}$ complementa que iniciativas como campanhas de mídia e vídeo são uma excelente forma de transmitir conhecimentos e práticas de segurança dos alimentos aos manipuladores, e podem alcançar um grande número de consumidores em suas casas.

Por meio do diagnóstico do perfil dos manipuladores em domicílios é possível conhecer o público alvo para desenvolver estratégias educativas com recursos direcionados e metodologia adaptada e específica, assim consegue-se atingir o objetivo da ação com máxima eficiência e mínimo esforço. Manipuladores domésticos que produzem refeições seguras são exemplos para seus filhos, promovendo um ambiente que reflete higiene e bons hábitos e, consequentemente, essas crianças serão adultos e profissionais mais conscientes e comprometidos.

\section{Conclusão}

Com base no que foi exposto, pode-se concluir que os manipuladores de alimentos nos domicílios pesquisados em Santa Maria (RS) são predominantemente do gênero feminino, possuem ensino fundamental completo ou incompleto e a maior parte obtém um rendimento mensal familiar maior que 3 salários mínimos.

Pode-se constatar que os responsáveis pela manipulação de alimentos nos domicílios são adultos e que não possuem outra função além dos afazeres domésticos. De maneira geral observou-se um baixo número de pessoas residindo nas casas, sendo verificado o hábito de realizar principalmente o almoço na residência.

Os participantes demonstraram não ter capacitação para a manipulação segura dos alimentos, assim como não vinculam as DTA ao consumo de alimentos no lar. Em contrapartida, mostrou-se que gostariam de aprender sobre o assunto, assim como foi verificado que a maioria dos entrevistados possui acesso a meios de comunicação.

Estudos desta magnitude dão subsídios para a aplicação mais coerente de ações educativas junto à população, pois acredita-se que a educação sanitária se inicia em casa, sendo complementada na escola. 


\section{Colaboradores}

BC Deon, LB Medeiros, LH Hecktheuer e ALF Saccol participaram igualmente de todas as etapas de elaboração do artigo.

\section{Referências}

1. Brasil. Ministério da Saúde (MS). Agência Nacional de Vigilância Sanitária. Resolução RDC no. 216, de 15 de setembro de 2004. Dispõe sobre regulamento técnico de Boas Práticas para serviços de alimentação. Diário Oficial da União 2004; 15 set.

2. Oliveira AM, Gonçalves MO, Shinohara NKS, Stamford TLM. Manipuladores de alimentos: um fator de risco. Hig Aliment 2003; 17(114/115):12-19.

3. Mendes RA, Coelho AIM, Azeredo RMC. Contaminação por Bacillus cereus em superfícies de equipamentos e utensílios em unidade de alimentação e nutrição. Cien Saude Colet 2011; 16(9):3933-3938.

4. Brasil. Ministério da Saúde (MS). Dados epidemiológicos - DTA período de 2000 a 2011. [página da Internet]. [acessado 2011 out 14]. Disponível em: http://portal.saude.gov.br/portal/arquivos/pdf/ 10_passos_para_investigacao_surtos.pd.

5. Europen Food Safety Authority (EFSA). The European Union Summary Reporton Trends and Sources of Zoonoses, Zoonotic Agents and food-borne outbreaks in 2009. J EFSA 2011; 9(3):378p.

6. Redmond EC, Griffith CJ. Consumer food handling at home: a review of food safety studies. J Food Prot 2003; 66(1):130-161.

7. Van Asselt ED, Jong AEI, Jonge R, Nauta MJ. Crosscontamination in the kitchen: estimation of transfer rates for cutting boards, hands and knives. $J$ Appl Microbiol 2008; 105(5):1392-1401.

8. Gilbert SE, Whyte R, Bayne G, Paulin SM, Lake RJ, van der Logt P. Survey of domestic food handling practices in New Zealand. Int J Food Microbiol 2007; 117(3):306-311.

9. Buccheri C, Mammina C, Giammanco S, Giammanco M, Guardia ML, Casuccio A. Knowledge, attitudes and self-reported practices of food service staff in nursing homes and long-term care facilities. Food Control 2010; 21(10):1367-1373.

10. Toku B, Ekuklu G, Berberoglu U, Bilge E, Dedeler $\mathrm{H}$. Knowledge, attitudes and self-reported practices of food service staff regarding food hygiene in Edirne, Turkey. Food Control 2009; 20(6):565-568.

11. Jevsnik M, Hlebec V, Raspor P. Food safety knowledge and practices among food handlers in Slovenia. Food Control 2008; 19(12):1107-1118.

12. Omemu AM, Aderoju ST. Food safety knowledge and practices of street food vendors in the city of Abeokuta, Nigeria. Food Control 2008; 19(4):396-402.

13. Meldrum R, Ribeiro CD, Simmons MD, Worthington D, Griffith C. The Welsh food microbiological forum and the All-Wales shopping basket sampling program: a model for the surveillance of microbiological quality in ready-to-eat foods. J Environ Health 2003; 65(10):24-28.

14. Sagoo SK, Little CL, Griffith CJ, Mitchell RT. Study of cleaning standards and practices in food premises in the United Kingdom. Commun Dis Public Health 2003; 6(1):6-17. 
15. Sanlier N. The knowledge and practice of food safety by young and adult consumers. Food Control 2009; 20(6):538-542.

16. Behrens JH, Barcellos MN, Frewer LJ, Nunes TP, Franco BDGM, Destro MT, Landgraf M. Consumer purchase habits and views on food safety: A Brazilian study. Food Control 2010; 21(7):963-969.

17. Sammarco ML, Ripabell G, Grasso GM. Consumer attitude and awareness towards food-related hygienic hazards. J Food Safety 1997; 17(4):215-221.

18. Oliveira MN, Brasil ALD, Taddei JAAC. Avaliação das condições higiênico-sanitárias das cozinhas de creches públicas e filantrópicas. Cien Saude Colet 2008; 13(3):1051-1060.

19. Redmond EC, Griffith CJ. Consumer perceptions of food safety risk, control and responsibility. Appetite 2004; 43(3):309-313.

20. Abdul-Mutalib N, Abdul-Rashid M, Mustafa S, Amin-Nordin S, Hamat RA, Osman M. Knowledge, attitude and practices regarding food hygiene and sanitation of food handlers in Kuala Pilah, Malaysia. Food Control 2012; 27(2):289-293.

21. Santa Maria. Lei complementar no 042 , de 29 de dezembro de 2006. Cria unidades urbanas, altera a divisão urbana de Santa Maria, dá nova denominação aos bairros e revoga a Lei Municipal no 2770/86, de 02/07/1986, Artigos $2^{\circ}$ a 25 e dá outras providências. Santa Maria, RS, 2006. [página da Internet]. [acessado 2011 out 25] Disponível em: http://www. santamaria.rs.gov.br/docs/secretarias/ArqSec60.pdf.

22. Losasso C, Cibin V, Cappa V, Roccato A, Vanzo A, Andrighetto I, Ricci A. Food safety and nutrition: Improving consumer behavior. Food Control 2012; 26(2):252-258.

23. Szabo M. The challenges of "re-engaging with food". Connecting employment, household patterns and gender relations to convenience food consumption in North America. Food, Culture \& Society 2011; 14(4):547-566.

24. Soares C, Sabóia AL. Tempo, trabalho e afazeres domésticos: um estudo com base nos dados da Pesquisa Nacional por Amostra de Domicílios de 2001 e 2005. Rio de Janeiro: IBGE; 2007.

25. Gong SL, Yang YS, HuaShen, Wang XY, Guo HP, Li Bai. Meat handling practices in households of Mainland China. Food Control 2011; 22(5):749-755.

26. Instituto Brasileiro de Geografia e Estatística (IBGE). Pesquisa de Orçamentos Familiares. 2009. [página da Internet]. [acessado 2012 jun 01]. Disponível em: http://www.ibge.gov.br/home/presidencia/ noticiasphp?idnoticia $=2084$.

27. Instituto Brasileiro de Geografia e Estatística (IBGE). Indicadores Sociais Municipais do Censo 2010. 2010. [página da Internet]. [acessado 2012 jun 9]. Disponível em: http://www.ibge.gov.br/home/presidencia/ noticias/noticia_visualiza.php?id_noticia $=2019 \&$ id_ pagina $=1$
28. Akutsu RC, Botelho RA, Camargo EB, Sávio KEO, Araújo WC. Adequação das Boas Práticas de Fabricação em Serviços de Alimentação. Rev Nutr 2005; 8(3):419-427.

29. Daniels S, Glorieux I, Minnen J, van Tienoven TP. More than preparing a meal? Concerning the meanings of home cooking. Appetite 2012; 58(3):1050-1056.

30. Wilcock A, Pun M, Khanona J, Aung M. Consumer attitudes, knowledge and behavior. A review of food safety issues. Trends Food Sci Technol 2004; 15(2):5666.

31. Shapiro MA, orticella N, Jiang LC, Gravani RB. Predicting intentions to adopt safe home food handling practices. Applying the theory of planned behavior. Appetite 2011; 56(1):96-103.

32. Leite LHM, Machado PAN, Vasconcellos ALR, Carvalho IM. Boas práticas de higiene e conservação de alimentos em cozinhas residenciais de usuários do programa saúde da família-Lapa. Rev Ciênc Méd 2009; 18(2):81-88.

33. Leite LHM, Waissmann W. Surtos de toxinfecções alimentares de origem domiciliar no Brasil de 20002002. Hig Aliment 2006; 20(147):56-59.

34. Welker CAD, Both JMC, Longaray SM, Haas, Soeiro MLT, Ramos RC. Análise microbiológica dos alimentos envolvidos em surtos de doenças transmitidas por alimentos (DTA) ocorridos no estado do Rio Grande do Sul, Brasil. R Bras Bioci 2010; 8(1):44-48.

35. Brasil. Secretaria de Vigilância em Saúde. Investigação de surto de botulismo alimentar em Coruripe/Al, em abril de 2009. Boletim eletrônico epidemiológico 2010; 10:1-4.

36. Brasil. Secretaria de Vigilância em Saúde. Investigação de surto alimentar, Paulista/Pernambuco, 2001. Boletim eletrônico epidemiológico 2002; 2:5-7.

37. Unusan N. Consumer food safety knowledge and practices at homes in Turkey. Food Control 2007; 18(1):45-51.

Artigo apresentado em 22/04/2013

Aprovado em 02/06/2013

Versão final apresentada em 10/06/2013 